\title{
X-RAY OPTICS FOR SYNCHROTRON RADIATION
}

\author{
C. Malgrange
}

Laboratoire de Minéralogie-Cristallographie, associé au CNRS, Universités Paris 6 and 7 4 pl. Jussieu, 75252 Paris Cedex 05, France

This paper presents the main optical devices used to prepare a beam from X-ray synchrotron source: monochromators, flat or curved in order to intercept a larger angular divergence at the sample, mirrors and, finally, optics for polarized X-ray experiments. Since X-ray optics is based either on total reflection or on diffraction by perfect crystals, the basic fundamental results of X-ray dynamical theory, which are necessary to understand the reasons why one device should be chosen rather than the other, are also presented.

PACS numbers: $61.10 . D p, 78.90 .+t$

\section{Introduction}

Every "optical" experiment, whatever its wavelength range is, consists of three distinct parts: a source, an optical system to prepare the beam and a detector. Here, the optical systems well-adapted to synchrotron sources will be discussed.

$\mathrm{X}$-ray synchrotron sources main specific properties are:

- intense white spectrum,

- small divergences but experiments are far from the source,

- specific polarization properties.

Most X-ray experiments require monochromatic waves with a more or less small band-pass. The selected wavelength is either kept constant during the experiments (diffraction experiments, small-angle scattering...) or scanned (extended $\mathrm{X}$-ray absorption fine structure or EXAFS, X-ray absorption near-edge structure or XANES) and then a large part of this lecture will deal with monochromators (Sec. 3).

Although synchrotron sources are very brilliant, one does not want to lose too much photons and then wants a beam narrow enough to be fully intercepted by the sample. The solution is then to focus the beam at the sample. If the focus is small enough it becomes a point-like probe for spatial-resolved experiments. Curved monochromators (Sec. 4) or curved mirrors (Sec. 5) are then used.

The content of Secs. 3 to 5 is detailed in several review papers [1-5].

Finally, the polarization properties of synchrotron sources give rise to a rapidly growing field of research; i.e. the dependence of X-ray absorption spectra on polarization, either linear or circular (Sec. 6). 
The necessary preparation of the beam is analogous to what has to be done with visible light experiments but, due to the smallness of $\mathrm{X}$-rays wavelengths, the optical components which have to be designed are quite different. The most important difference between visible light and X-rays concerns the index of refraction which, for X-rays, is nearly equal to 1 . It can be expressed as $n=1-\delta$, where $\delta$ is of the order of $10^{-4}$ to $10^{-5}$ for most materials and then lenses do not exist for X-rays. There are two ways to go around this difficulty. The index of refraction being slightly less than 1 , the phenomenon of total reflection occurs for small enough glancing angles, typical a few tenths of a degree, and then one can prepare mirrors which can be used to focus $\mathrm{X}$-rays. The other important property of X-rays is Bragg reflection by crystals which is used to select pure wavelengths. The selected wavelength $\lambda$ is given by Bragg's law

$$
2 d_{h, k, l} \sin \theta=n \lambda,
$$

where $d_{h, k, l}$ is the spacing between the lattice planes of a given family $(h, k, l)$, normal to the reciprocal lattice vector $h[h, k, l]^{*} ; \theta$ is the angle between the incident $\mathrm{X}$-ray beam and the considered lattice planes and $n$ is an integer. This equation may also be written

$$
2 d_{n h, n k, n l} \sin \theta_{\mathrm{B}}=\lambda \text { since } d_{n h, n k, n l}=\frac{d_{h, k, l}}{n},
$$

which means that for a given value of $\theta$, i.e. a given position of the crystal with respect to the incident beam, not only wavelength $\lambda^{\prime}=n \lambda$ but also $\lambda^{\prime} / 2, \ldots \lambda^{\prime} / n$ called the harmonics are reflected corresponding respectively to diffraction vectors $h, 2 h, \ldots n h$ as far as these reflections are not forbidden by the crystal structure. The narrowest selected band-pass is obtained with perfect crystals. In such perfect crystals the optics is governed by $\mathrm{X}$-ray dynamical theory whose main results need to be recalled now.

\section{Some results of X-ray dynamical theory}

The classical kinematical theory of diffraction valid for ordinary crystals relies on the assumption that the diffracted intensity is very low compared to the incident intensity. This is no more valid in perfect crystals and one cannot treat separately the incident and diffracted waves which are then coupled giving rise to specific properties of diffraction.

For a given wavelength, a perfect crystal diffracts over a narrow angular range of the order of the arc second which is determined by the width of the so-called rocking curve (Fig. 1) which gives the reflecting power $R(\theta)$, ratio of the diffracted to the incident intensity as a function of the angle of incidence of the incident wave assumed to be a monochromatic plane wave. Before giving the value of this width it is necessary to define some geometrical parameters involved in Bragg diffraction. Figure 2 distinguishes between transmission or Laue case and reflection or Bragg case. Most monochromators are used in Bragg geometry and then, unless specified, Bragg geometry will be considered here. The angle of asymmetry $\alpha$ is defined, in Bragg geometry, as the angle between the crystal surface and the reflecting planes. 


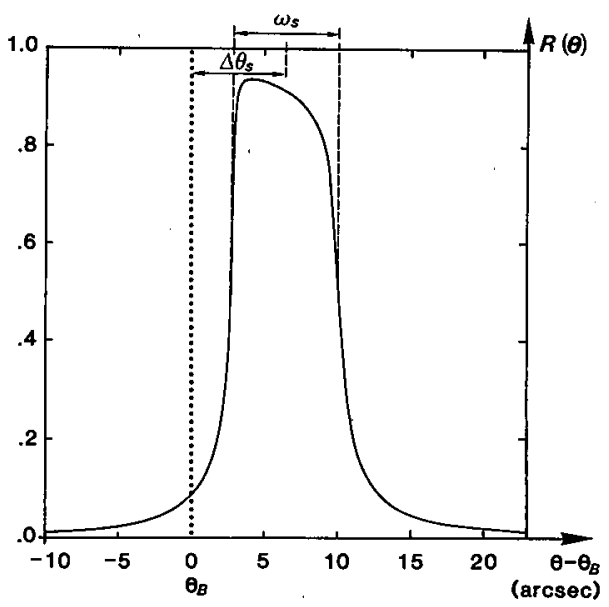

Fig. 1. Full line: $\mathrm{Si}(111)$ rocking curve calculated for $\lambda=1.55 \AA(E=8 \mathrm{keV})$ and the symmetric case. Dotted line: the same rocking curve assuming absorption to be zero.

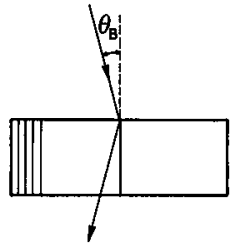

a

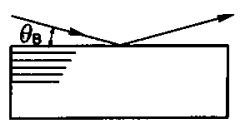

c
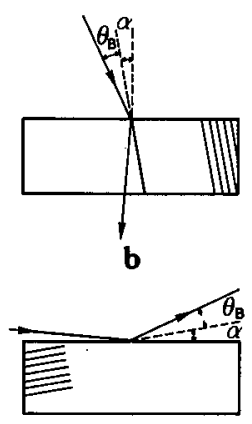

d

Fig. 2. Different diffraction geometries: (a) and (b) - transmission or Laue geometry, symmetric (a) and asymmetric (b) case; (c) and (d) - reflection or Bragg geometry, symmetric (c) and asymmetric (d) case.

For non-absorbing crystals the rocking curve (Fig. 1) presents a domain of total reflection whose angular width is

$$
\omega_{0}=\omega_{\mathrm{s}} / \sqrt{b} \text { where } b=\frac{\sin \left(\theta_{\mathrm{B}}-\alpha\right)}{\sin \left(\theta_{\mathrm{B}}+\alpha\right)}
$$

is the asymmetry factor and $\omega_{\mathbf{s}}$, which refers to the symmetric case and is called the Darwin width, is such that

$$
\omega_{\mathrm{s}}=\omega_{\mathrm{D}} \tan \theta_{\mathrm{B}} \quad \text { with } \omega_{\mathrm{D}}=\frac{4 r_{\mathrm{e}} d_{h k l}^{2} F_{h} \mathrm{e}^{-M}|C|}{\pi V} .
$$

$r_{\mathrm{e}}$ is the classical radius of electron, $V$ - the volume of the unit cell, $F_{h}-$ the structure factor, $\mathrm{e}^{-M}-$ the Debye-Waller factor which takes into account the 
thermal motion of the atoms in the crystal, $C$ - the polarization factor equal to 1 or $\cos 2 \theta$ if the polarization vector is respectively perpendicular or parallel to the diffraction plane hereafter preferably called scattering plane (in order not to make any confusion with the diffracting or reflecting planes) defined as the plane formed by the incident and diffracted directions.

Since $\omega_{0}$ varies as the square of $d_{h k l}$, the widths of the different harmonics decrease rapidly with the order of the harmonics (Fig. 3).

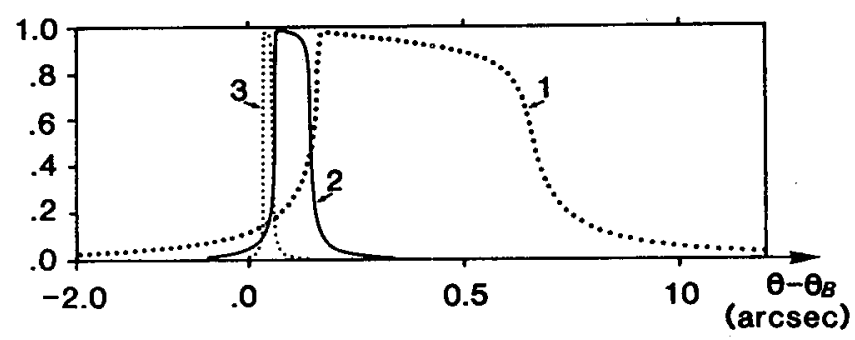

Fig. 3. Rocking curves for the different harmonics $\theta_{B}=23.6^{\circ}$. Curve $1: \lambda=1.54 \AA$, 220 reflection; curve $2: \lambda=0.77 \AA, 440$ reflection; curve $3: \lambda=0.513 \AA, 660$ reflection.

The middle of the domain of total reflection $\theta_{0}$ is shifted from the Bragg angle $\theta_{\mathrm{B}}$, defined by Eq. (2), by $\Delta \theta_{0}=\theta_{0}-\theta_{\mathrm{B}}=(1 / 2)(1+1 / b) \Delta \theta_{\mathrm{s}}$, where $\Delta \theta_{\mathrm{s}}=\left(\omega_{\mathrm{s}} / 2\right)\left(F_{0} / F_{h} \mathrm{e}^{-M}\right)$ is the shift for the symmetric case (Fig. 1).

For real crystals, absorption has to be taken into account and there is no more total reflection but a domain of quasi-total reflection, the width of which is of the order of $\omega_{0} . \omega_{0}$ is then commonly considered as the width of the rocking curve or the domain of angular acceptance of the crystal for the considered reflection. The divergence $\omega_{h}$ of the reflected beam is such that (see Fig. 4):

$$
\omega_{h}=\omega_{\mathrm{s}} \sqrt{b}
$$

If a white beam falls on a perfect crystal, the spectral band-pass depends on the angular divergence of the beam. To determine this band-pass it is convenient to introduce a graphic representation due to DuMond [6] which gives the correlation between the direction $\theta$ of the incident wave in the scattering plane (abscissa) and the wavelength $\lambda$ (ordinate). Figure 5 shows the $(\theta, \lambda)$ domain of the incident beam which is reflected when the angular divergence of the incident beam is equal to $\Omega$. For a symmetric reflection, the correlation between direction and wavelength in the incident and diffracted beams are the same. When such a reflected beam falls on a second crystal, two different settings have to be considered depending on the sign of the angle between the incident and reflected beams on the two successive crystals. If these signs are identical the setup is noted $(+,+)$, if they are different it is noted $(+,-)$. Figures $6 \mathrm{a}$ and $6 \mathrm{~b}$ show how the emerging domains are determined in these two cases. The position of the first crystal with respect to the incident beam determines the position of the beam diffracted by the first crystal (vertically hatched area) with respect to curve $1\left(\lambda=2 d_{1} \sin \theta\right)$, i.e. the wavelength domain issued from the first crystal. When the second crystal is rotated with respect to 


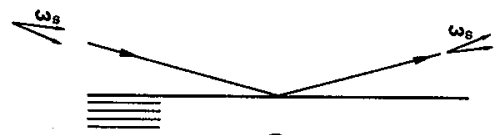

(a)
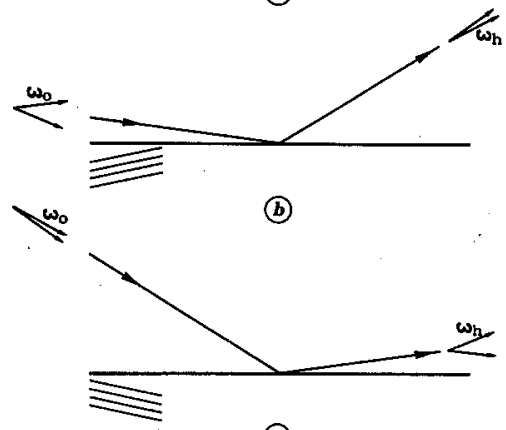

(c)

Fig. 4. (a) Symmetric case; the angular widths of the domain of quasi-total reflection in incident $\left(\omega_{0}\right)$ and reflected $\left(\omega_{h}\right)$ beams are equal: $\omega_{0}=\omega_{h}=\omega_{s} ;(b)$ and (c) asymmetric cases; for (b) geometry $(\alpha>0) \omega_{0}$ is larger than $\omega_{s}$ and $\omega_{h}$ smaller. Such an asymmetry is used to obtain a nearly plane wave.

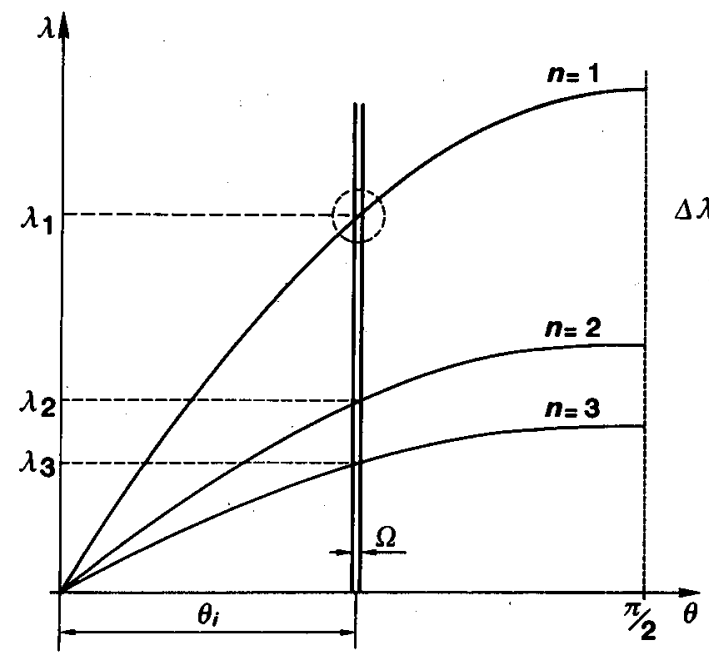

(a)

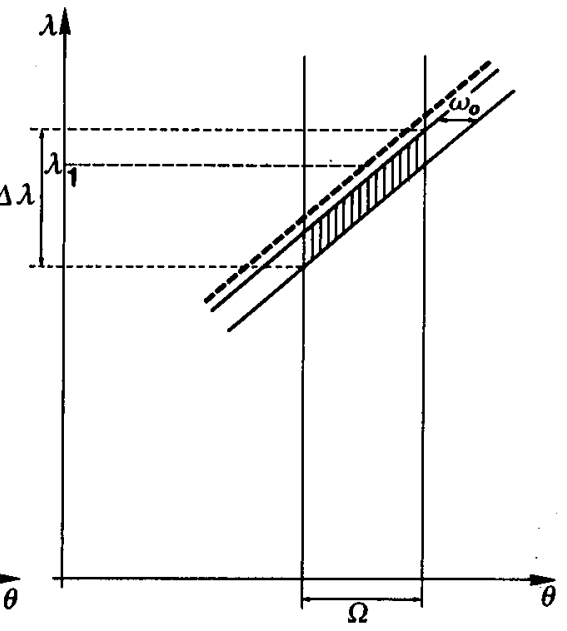

(b)

Fig. 5. DuMond diagram: the white incident beam has a divergence $\Omega$. The mean angle of incidence on lattice planes $(h, k, l)$ is $\theta_{i}$. (a) Fundamental wave length $\lambda_{1}$ and harmonics $\left(\lambda_{2}, \lambda_{3} \ldots\right)$ are diffracted. (b) Magnification of the spectral width $\Delta \lambda$ around $\lambda_{1}$. The dotted line is the curve $\lambda=2 d_{h k l} \sin \theta$. The domain of quasi-total reflection is slightly shifted with respect to the dotted line which results in a slight shift of the mean wavelength with respect to $\lambda_{1}$. 


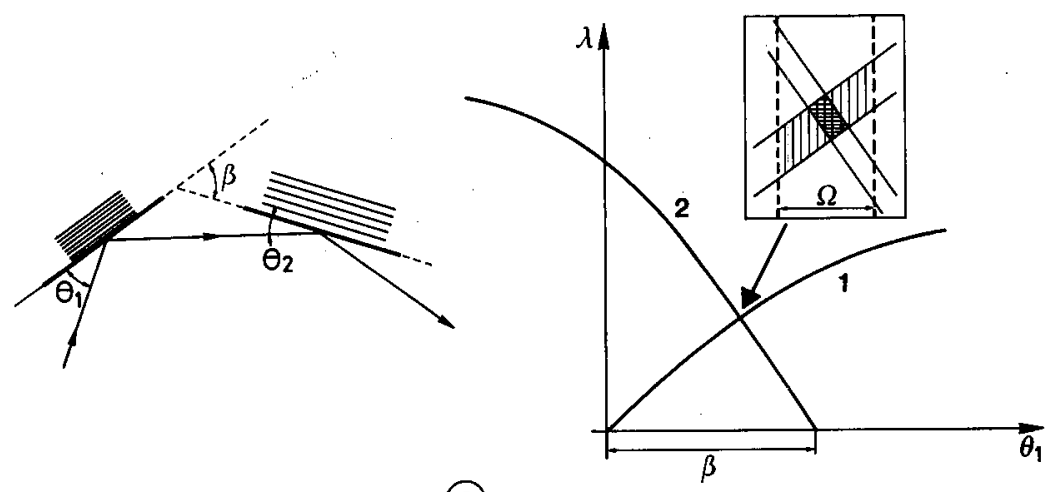

(a)
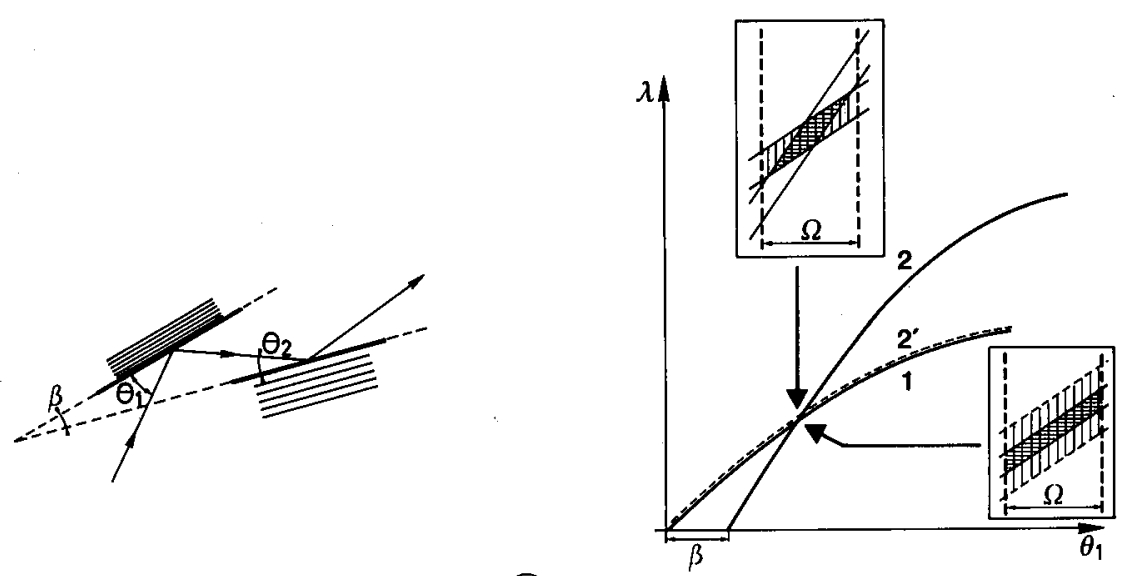

(b)

Fig. 6. Two different setups and the corresponding DuMond diagrams. (a) $(+,+)$ setup. (b) $(+,-)$ setup where curves 1 and 2 correspond to different lattice parameters $d_{1}$ and $d_{2}$, curves 1 and $2^{\prime}$ to identical lattice parameters. In the latter case the first crystal is assumed to be asymmetrically cut (the same geometry as in Fig. $4 \mathrm{~b}$ ) and the second crystal to be symmetric (lower insert).

the first one, curve 2 is translated with respect to curve 1 . In the $(+,+)$ setup, diffraction by crystal 2 takes place over an angular rotation roughly equal to the divergence $\Omega$ of the incident beam. For each position of crystal 2, the wavelength band-pass is small and the mean wavelength varies during the rotation: the setup is dispersive. In the $(+,-)$ setup and if the lattice parameters of both crystals are exactly the same, curves 1 and 2 are parallel. The wavelength band-pass is larger than in the dispersive setup but practically does not vary during the very small rotation where diffraction takes place. Such a setup is a non-dispersive parallel one. 


\section{Flat crystal monochromators}

In order to obtain a monochromatized beam whose direction does not depend on the selected wavelength the simplest monochromator consists of two identical and parallel crystals in the $(+,-)$ non-dispersive set-up which are turned together when a wavelength scan is required as in EXAFS experiments for example.

If $R_{\lambda}(\theta)$ is the reflecting power for one reflection and a given wavelength, the integrated emerging intensity is

$$
I(\lambda)=\int R_{\lambda}(\theta) R_{\lambda}(\theta-\beta) \mathrm{d} \theta=\int_{\theta_{1}}^{\theta_{2}} \boldsymbol{R}_{\lambda}(\theta, \beta) \mathrm{d} \theta,
$$

where $\theta_{1}=\theta_{i}-\Omega / 2, \theta_{2}=\theta_{i}+\Omega / 2, \theta_{i}$ being the mean angle of incidence and $\Omega$ the angular divergence of the incident beam. The maximum intensity is obtained if $\beta$ is equal to zero and is then equal to

$$
\int \boldsymbol{R}_{\lambda}(\theta, \beta=0) \mathrm{d} \theta=\int R_{\lambda}(\theta)^{2} \mathrm{~d} \theta .
$$

It is easily shown that the variation of $I(\lambda)$ as a function of $\lambda$ is related to the convolution of the curve $\boldsymbol{R}_{\lambda}(\theta, \beta=0)$ by the intensity profile of the incident beam $I_{0}(\theta)$, the variables $\theta$ and $\lambda$ being related by $\Delta \lambda / \lambda=\Delta \theta \cot \theta$. Then the energy band width can be approximated by

$$
\Delta \lambda / \lambda=\Delta E / E=\left(\omega^{2}+\Omega^{2}\right)^{1 / 2} \cot \theta_{\mathrm{B}}
$$

where $\omega$ is the width of the curve $\boldsymbol{R}_{\lambda}(\theta, \beta=0)$, i.e. at a good approximation the width $\omega_{0}$ of the rocking curve and $\Omega$ the angular width of the incident beam in the diffracting plane.

It should be pointed out here that the tails of the rocking curve induce tails in the curve $I(\lambda)$. Successive reflections decrease tails in $\boldsymbol{R}_{\lambda}(\theta)$ and then in $I(\lambda)$.

Due to the narrow width of the rocking curves a slight misorientation $\beta$ of the two crystals decreases the width of $\boldsymbol{R}_{\lambda}(\theta, \beta)$ (which can be useful in some specific experiments) but decreases also the intensity of the emerging beam which can even become null for a too large misorientation $\beta$. The same effect occurs for a slight variation of parameter which can be induced for example by a difference of temperature (as described below).

a) Channel-cut monochromators: the easiest solution to avoid misorientation between the two crystals is to use an U-shaped monolithic crystal obtained by cutting a slot in a single crystal. Then the emerging beam although parallel to the incident beam is slightly shifted (Fig. 7a) which implies either to change the sample position between two experiments at two different wavelengths or to have samples large enough to intercept the beam over the considered shift, and homogeneous. Furthermore, with second or third generation of synchrotron sources the thermal load on the first part of the block induces a temperature difference and then a variation of lattice parameters between the two parts which may cancel the emerging intensity.

b) Two separated crystal-monochromators: if the two crystals are separated, a fixed exit monochromatic beam can be obtained by means of a single axis rotational stage and a device (for example a cam in [7]) which translates as necessary the first or the second crystal. 

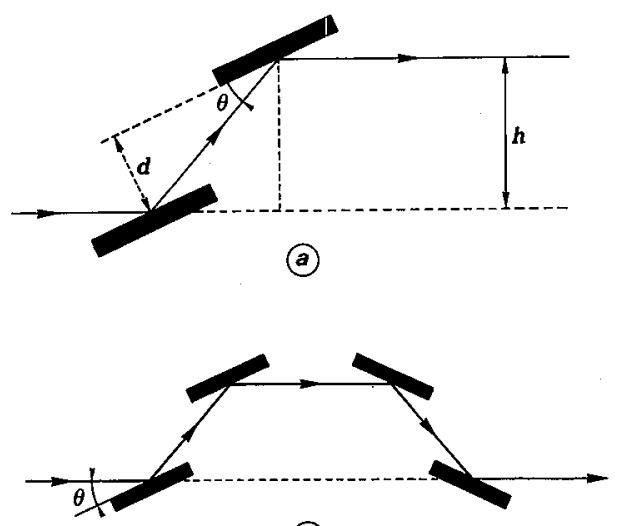

(b)

Fig. 7. Different types of monochromator crystal arrangements. (a) Two crystal non-dispersive arrangement: the shift $h$ of the beam, equal to $2 d \cos \theta_{\mathrm{B}}$, varies with wavelength. If the two crystals are separated a fixed exit of the beam can be obtained if the second crystal is translated as necessary during the scan. (b) Four-crystal dispersive arrangement which keeps a fixed exit.

c) Four-crystal monochromators [8, 9]: the four crystals are identical and adjusted for the same reflections; the two first parallel crystals form a non-dispersive pair which is arranged in a dispersive mode with respect to the two last parallel crystals (Fig. $7 \mathrm{~b}$ ). In order to scan the wavelength the two pairs of crystals are counter rotated of the same angle. The beam has a fixed exit and the energy resolution which does not depend on the divergence of the incident beam is better than in cases (a) and (b). It can be approximated to

$$
\Delta \lambda / \lambda=\Delta E / E=\left(\omega_{\mathrm{s}} / 2\right) \cot \theta \text {. }
$$

d) Very small band-pass monochromators. For some experiments like standing-wave experiments (XSW) or plane-wave topography or diffractometry the incident beam must have a divergence smaller than the width of the rocking curve of the investigated sample. The most common solution uses for the monochromator and the sample a non-dispersive parallel setup; the width of the wave emerging from the monochromator is reduced by designing a monochromator with successive reflexions with either one asymmetric reflexion [1] or a slight detuning between two symmetric reflexions [10]. These two setups permit also to eliminate the harmonics (Fig. 8 taken from [1]).

Another possibility is to design a monolithic silicon crystal with three successive reflexions where the two first are in a dispersive $(+m,+n)$ setup defining the wavelength and the angular and spectral width and the two last ones are asymmetric non-dispersive $(+n,-n)$ reflections with different asymmetries in order to suppress the harmonics and reduce again the angular and spectral width [11]. Such a setup provides a beam with a very narrow angular and spectral width, but is not tuneable. 


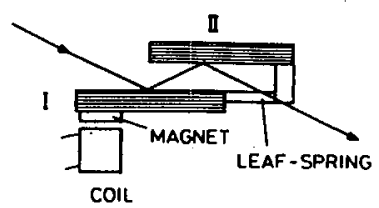

a
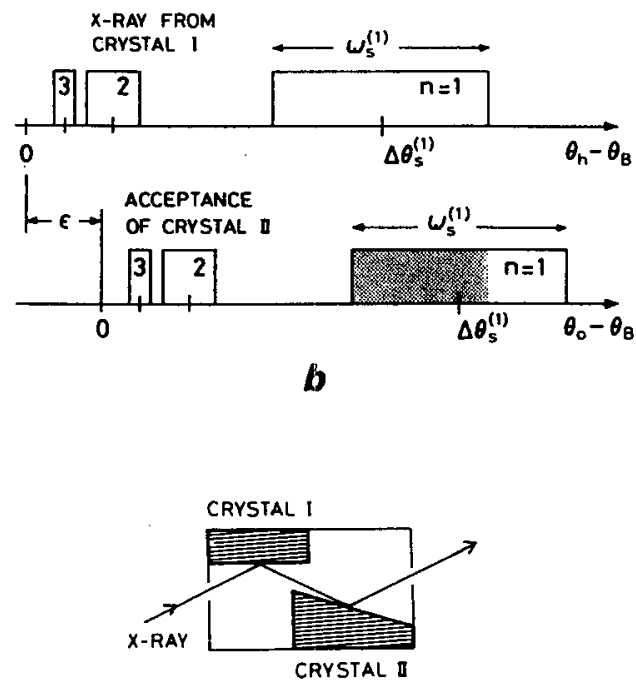

C
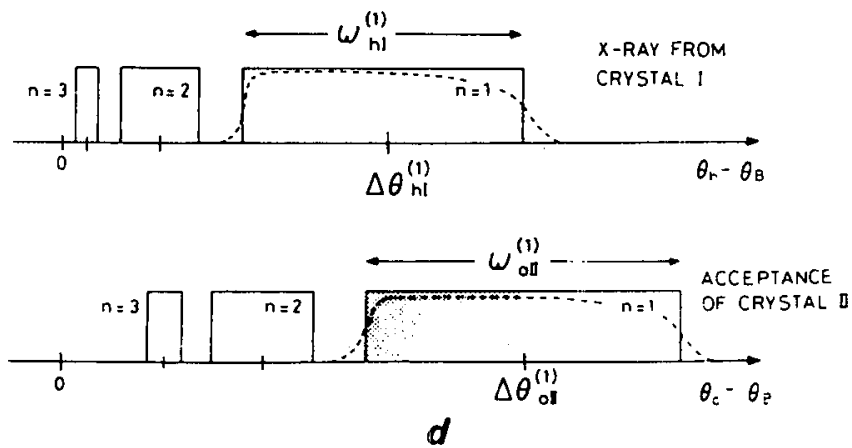

Fig. 8. An offset harmonics rejection monochromator (a) schematic view. (b) Principle of harmonics rejection. The rocking curves are supposed to be top-hat functions. The offset $\varepsilon$ is smaller than the Darwin width $\omega_{s}^{(1)}$ of the fundamental wavelength and larger than those of harmonics. (c) Monolithic harmonics rejection monochromator. Crystals I and II have different angles of asymmetry inducing different shifts of the rocking curves with respect to the Bragg angle $\theta_{\mathrm{B}}$. These differences are large enough for harmonics to be rejected but fundamental wavelength is reflected (shaded area) (d). After [1]. 


\section{Curved crystal optics}

\subsection{Curved monochromators}

Because of large distances between the synchrotron radiation source and the experiments, condensing optics are useful to confine the beams to a small size at the sample and to collect as much of the radiation as possible.

Two types of optics can be used based on either specular reflection (mirrors) or Bragg diffraction (curved crystals). The second technique is first discussed here.

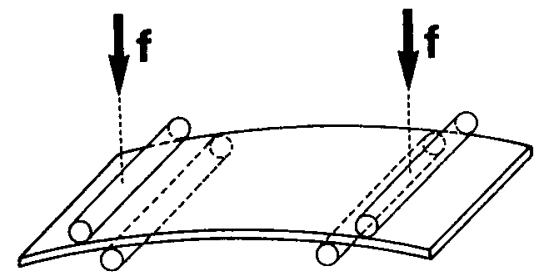

(a)

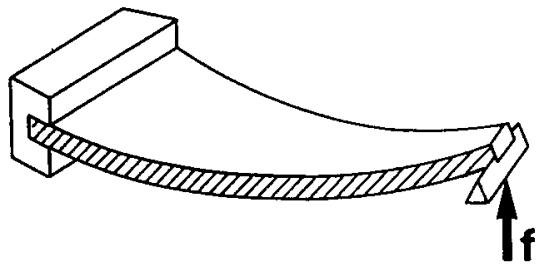

(b)

Fig. 9. Two types of crystal benders: (a) four-cylinder crystal bender; (b) triangular crystal.

Two main types of bending devices were used (Fig. 9):

- the four-cylinder mechanism to bend rectangular plates [12-14],

- a triangular crystal whose basis is clamped and a screw-driven force is applied to the apex $[15,16]$.

Let us suppose, as an example, that one wants to condense the horizontal divergence. There are then two different cases depending on if the scattering plane is vertical (the focusing is then "saggital", i.e. the curvature is perpendicular to the scattering plane) or horizontal (the focusing is then "meridional", i.e. the curvature is parallel to the scattering plane).

\subsubsection{Saggital focusing}

Let us consider a two-crystal monochromator whose first crystal is a flat crystal diffracting in a vertical plane. This crystal and the vertical divergence determine the wavelength resolution. Let us suppose first that the second crystal is flat. The rays impinging the second crystal seem to come from a virtual source $S$ symmetric of the synchrotron source with respect to the first crystal. A ray diffracted in the vertical plane follows the path $S M F$ (Fig. 10). Let us consider a ray which makes, in the "horizontal plane" (in fact in the plane symmetric of the horizontal plane with respect to the first crystal) an angle $\psi$ with $S M$. Its angle of incidence $\theta^{\prime}$ on the crystal is slightly different from $\theta$ :

$$
\theta^{\prime}-\theta=\varepsilon=\tan \theta\left(\psi^{2} / 2\right)
$$

and its wavelength is slightly different from the one of $S M$.

Since its scattering plane makes an angle $\psi$ with the vertical one, at a distance $p+q$ from the source ( $p$ being the distance source-monochromator and $q$ 


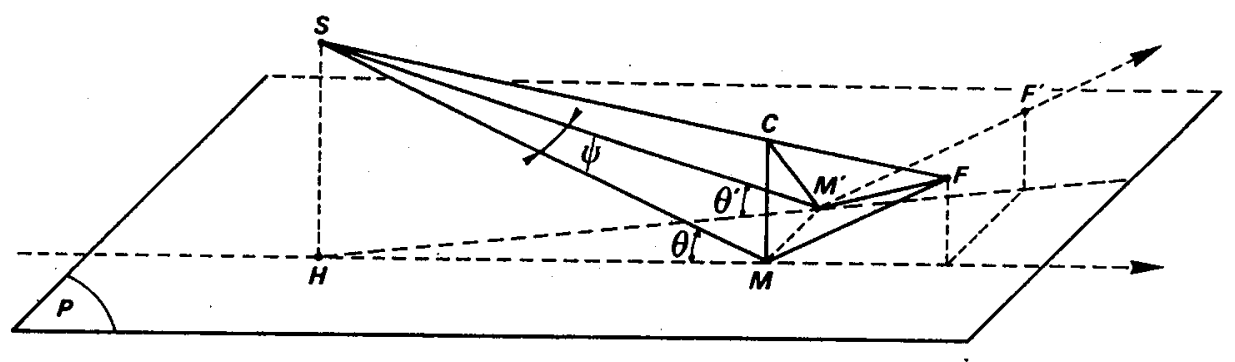

Fig. 10. Principle of saggital curvature.

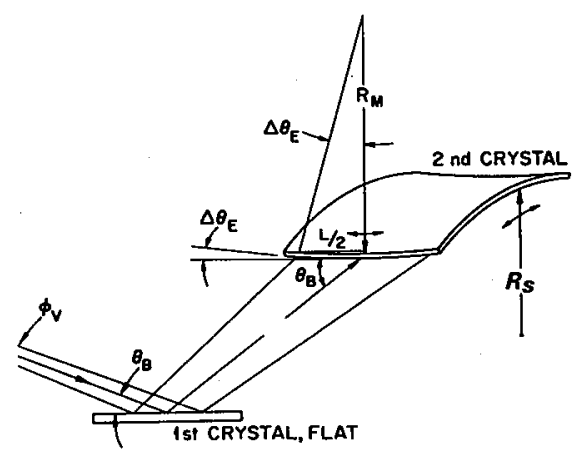

Fig. 11. An anticlastic curvature $R_{\mathrm{M}}$ is generated when a thin plate is bent to the radius $R_{S}$ and results in a variation of angle of incidence $\Delta \theta_{\mathbf{E}}$ for meridian rays divergent from the central ray. After [17].

the distance monochromator-sample) it will be shifted laterally from $F$ to $F^{\prime}$ by $(p+q) \psi$ and probably will be outside the sample and then lost for the experiment.

In order to make this beam follow the path $S M^{\prime} F$ the diffraction vector $h$ in $M^{\prime}$ should be in this plane and parallel to $M^{\prime} C$. The crystal should be curved with a radius $C M=(2 p q \sin \theta) /(p+q)$.

Once the crystal is curved, the angle of incidence of $S M^{\prime}$ is no more exactly $\theta^{\prime}$ but $\theta^{\prime}+\varepsilon^{\prime}$. In order the beam to be still diffracted by the crystal, $\varepsilon^{\prime}$ should be less than the Darwin width. It was shown [17] that this condition is fulfilled for a larger range of horizontal divergence if the ratio $q / p$ is equal to $1 / 3$. The ratio $q / p$ is also the magnification $m$ (the image of the source is $m$ times the size of the source). In fact, the ideal surface for the crystal should be a fraction of an ellipsoide of revolution around $S F$. The cylindrical curvature discussed here is tangent to the ellipsoid; it is a good approximation and still better if the magnification $m$ is equal to $1 / 3$. It was shown that in order not to be at $m=1 / 3$ and to focus a large divergence one could use a conical surface.

In fact, pure cylindrical bending of even thin rectangular plates results in a transverse curvature of the plate called anticlastic curvature which results in a variation of the angle of incidence $\Delta \theta_{\mathrm{E}}$ for meridian rays divergent from the 


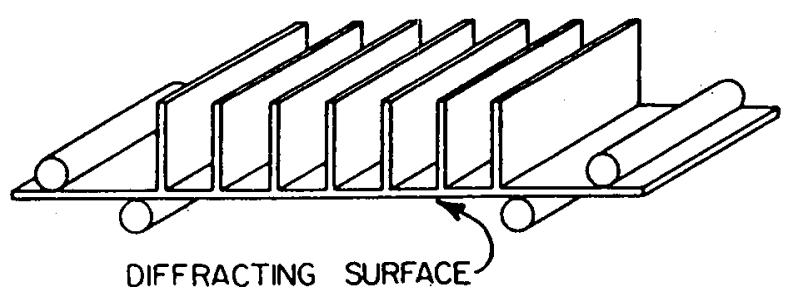

Fig. 12. Schematic view of a monolithic crystal with ribs cut parallel to the bending rods to prevent anticlastic bending.

central ray (Fig. 11). The anticlastic radius of curvature $R_{\mathrm{M}}$ is related to the sagittal radius of curvature $R_{\mathrm{S}}$ by the relation $R_{\mathrm{M}}=R_{\mathrm{S}} / \nu$, where $\nu$ is Poisson's ratio and the crystal is assumed to be isotropic. The influence of anisotropy is studied in [18].

In order to reduce the anticlastic curvature, it is possible to attach to the back surface transverse ribs, but the glue causes appreciable distortions in the crystallographic planes. To eliminate these distortions the ribs and the thin crystal are cut in a thick monolithic Si block (Fig. 12) [12].

\subsubsection{Meridional focusing}

Whereas the anticlastic bending has to be strongly reduced for saggital focusing (because it induces immediately a variation of the angle of incidence on the diffracting planes), with meridional focusing, the anticlastic bending has a negligeable influence.

In order to obtain a focused monochromatic diffracted beam the distances source-crystal $p$, crystal-focus $q$ and the radius of curvature $R$ should be related by the relations

$$
\begin{aligned}
& \frac{2}{R}=\frac{\sin (\theta-\alpha)}{p}+\frac{\sin (\theta+\alpha)}{q}, \\
& \frac{p}{q}=\frac{\sin (\theta-\alpha)}{\sin (\theta+\alpha)}
\end{aligned}
$$

where $\alpha$ is the angle of asymmetry. Equation (8) yields for the focusing and Eq. (9) for a monochromatic focusing.

The usual setup is the Johann geometry [19] (Fig. 13) which induces spherical aberration. The focusing would be perfect in the Johansson geometry where the monochromator surface is grinded in order to be curved with a radius of curvature equal to $R$ when unstressed and then homogeneously curved with a radius equal to $R / 2$. Then the radius of the diffracting planes is equal to $R$ and that of the surface of the crystal is equal to $R / 2$, i.e. the radius of the so-called Rowland circle on which are located the source, the crystal and the focus. Such a setup is not easily tuneable since the radius of curvature is predetermined by that of the unstressed surface of the crystal. Another way to eliminate the spherical aberration is to have an ellipsoidal surface with the source and the focusing point at the focuses of the 


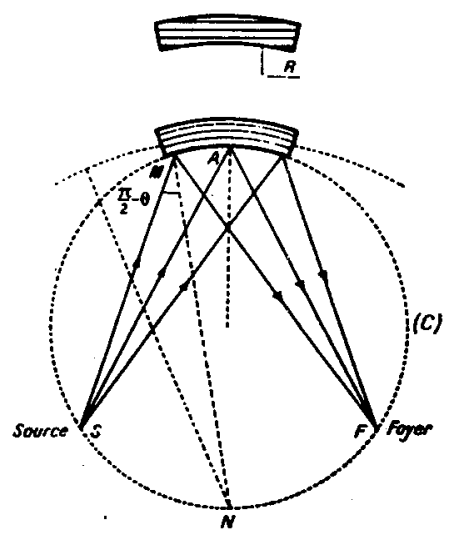

(a)

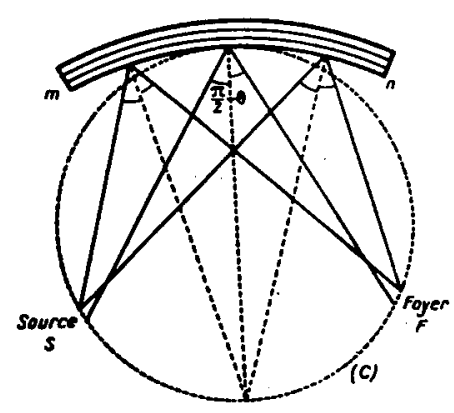

(b)

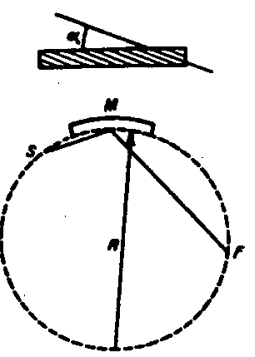

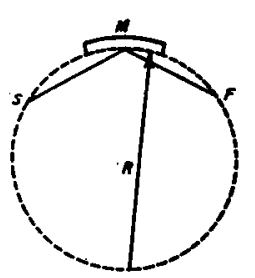

(C)

Fig. 13. Reflection focusing geometries for curved crystals. (a) Johansson geometry. Rays issued from $S$ focus in $F$. The dotted circle is Rowland circle whose diameter is equal to the radius of curvature $R$ of the bent lattice planes. (b) Johann geometry. Focusing is not perfect because the surface of the crystal does not follow the Rowland circle exactly. (c) Dependence of geometrical parameters $p$ (distance source-crystal) and $q$ (distance crystal-focus) on the angle of asymmetry. See formulas (8) and (9).

ellipsoid and an elliptical cylinder is a good approximation. Such a curvature can be obtained with a triangle profiled along an adequate shape [16].

\subsection{Polychromatic focalization; application to dispersive XAFS}

Let us consider a meridional focusing such that only condition (8) is satisfied. The variation $\theta_{2}-\theta_{1}$ of angle of incidence between the two extremities of the curved crystal is given by

$$
\theta_{2}-\theta_{1}=\frac{L}{2}\left(\frac{\sin (\theta+\alpha)}{q}-\frac{\sin (\theta-\alpha)}{p}\right)
$$


where $L$ is the length of the crystal (Fig. 14).

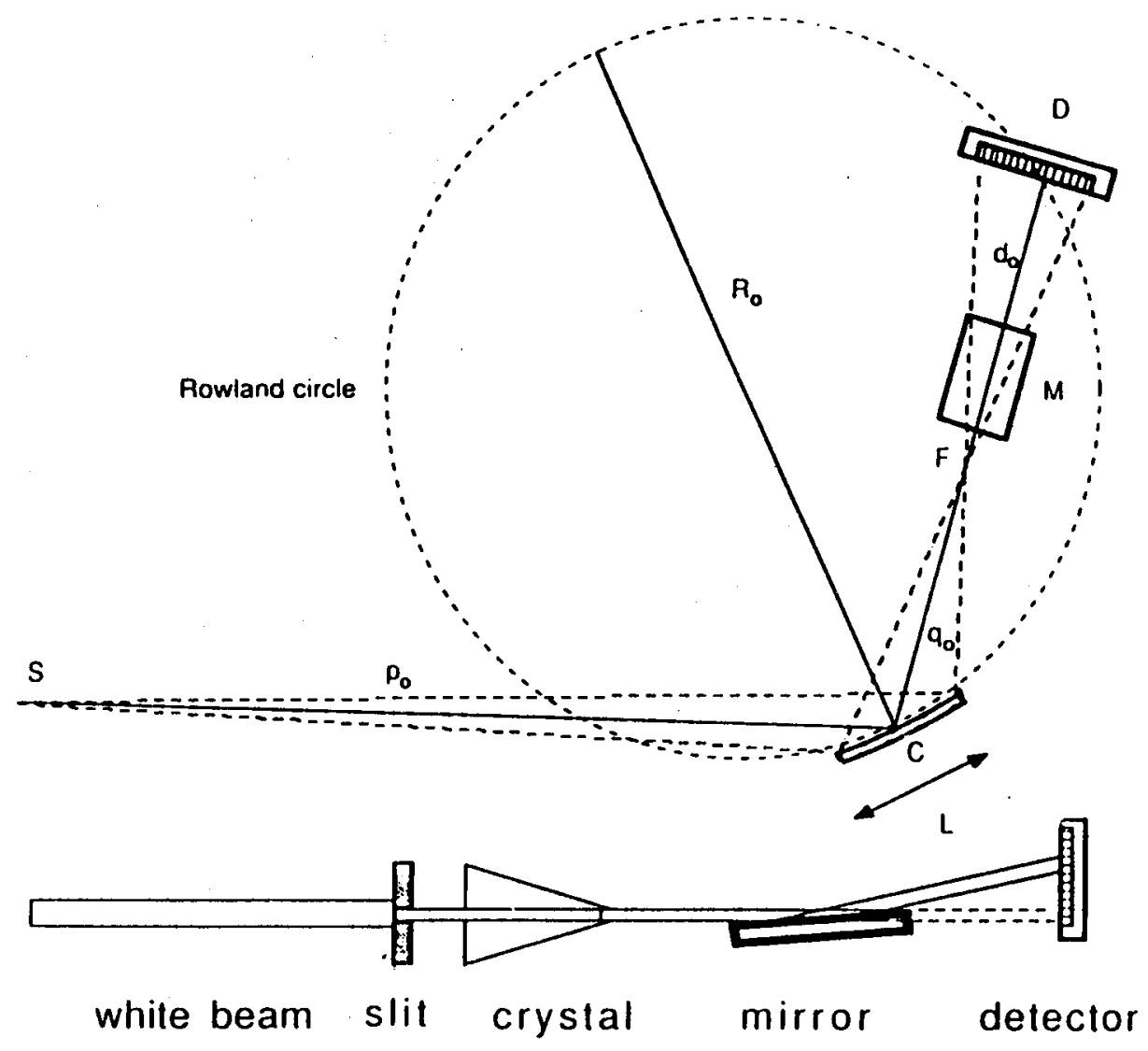

Fig. 14. Geometry of the focusing time-resolved XAS spectrometer. Polychromatic focusing in $F$ by a bent single crystal $(C)$. The position sensitive detector $(D)$ is located on the Rowland circle where monochromatic focusing (necessary because of the finite size of the source) occurs. A fused quartz mirror $(M)$ ensures harmonic rejection. After [16].

For a point source $S$, the polychromatic beam focuses in $F$ and diverges after. A linear detector placed beyond $F$ analyses the beam as a function of $\lambda$. Such a setup was developed at the synchrotron facility in Orsay (France) to obtain at once all the XAFS (X-ray absorption fine structure) spectra given by a sample put at the focus and to do time-resolved experiments [16]. A mirror is placed between the sample and the detector in order to eliminate the harmonics (see below). 


\section{Mirrors}

The refraction index for X-rays can be written

$$
n=1-\delta \quad \text { with } \delta=\frac{\lambda^{2} r_{\mathrm{e}}}{2 \pi} \sum N_{j} f_{j}
$$

$r_{\mathrm{e}}$ is the classical radius of electron, $\lambda$ - the wavelength, $N_{j}$ is the number of atoms of species $j$ per unit volume, $f_{j}$ - the atomic scattering factor in incident direction which is equal to

$$
f_{j}=Z_{j}+\Delta f_{j}^{\prime}+\mathrm{i} \Delta f_{j}^{\prime \prime}
$$

where $Z_{j}$ is the atomic number of $j$-th atom, $\Delta f_{j}^{\prime}$ and $\Delta f_{j}^{\prime \prime}$ the corrections for anomalous dispersion; $\Delta f_{j}^{\prime \prime}$ is related to the atomic absorption coefficient or atomic cross-section for absorption $\mu_{\mathrm{a}}^{j}$ by the relation

$$
\mu_{\mathrm{a}}^{j}=2 \lambda r_{\mathrm{e}} \Delta f_{j}^{\prime \prime} \text {. }
$$

Since the real part of $n$ is less than 1 there exists specular reflection for grazing angles $\theta$ less than a critical angle $\theta_{\mathrm{c}}$ such that

$$
\cos \theta_{\mathrm{c}}=1-\delta_{\mathrm{r}}
$$

where $\delta_{\mathrm{r}}$ is the real part of $\delta$. Since $\delta_{\mathrm{r}}$ is very small and neglecting the anomalous dispersion,

$$
\theta_{\mathrm{c}}=(2 \delta)^{1 / 2}=\lambda\left(r_{\mathrm{e}} \mathcal{N} / \pi\right)^{1 / 2},
$$

where $\mathcal{N}$ is the number of electrons per unit volume. $\theta_{c}$ increases with $\mathcal{N}$ and then is larger for mirror surfaces coated with heavier elements and increases proportionally to $\lambda$. As an example for a gold coated mirror and $\lambda=1.54 \AA(E \cong 8 \mathrm{keV})$, $\theta_{\mathrm{c}}=10 \mathrm{mrad} \cong 0.5^{\circ}$ whereas for $\lambda=15.4 \AA(E \cong 800 \mathrm{eV}), \theta_{\mathrm{c}} \cong 5^{\circ}$. Corresponding values for silica are divided by 3 .

For a beam incident on a mirror with an angle $\theta_{0}$, wavelengths with a critical angle less than $\theta_{0}$ are reflected, i.e. wavelengths $\lambda$ larger than $\lambda_{0}=\theta_{0}\left(\pi / r_{\mathrm{e}} \mathcal{N}\right)^{1 / 2}$. Figure 15 shows the reflectivity of a platinum coated glass mirror for various angles of incidence [21]. The cut-off angle is not sharp because of absorption. Mirrors act then as filters which suppress high-energy photons and they are used to eliminate higher harmonics of Bragg reflections from crystal monochromators.

Mirrors can be polished to obtain curved surfaces and such curved mirrors are used to focus $\mathrm{X}$-ray beams. At X-ray energies, $\theta_{c}$ values are typically a fraction of a degree and then mirrors need to be long, of the order of 1 metre (for example at an incidence of $3 \mathrm{mrad}$ a $1 \mathrm{~m}$ mirror placed at $20 \mathrm{~m}$ from the source would intercept $0.15 \mathrm{mrad}=0.5$ minute) which is an advantage as far as heat loading is concerned but a disadvantage with respect to the cost. The efficiency of focusing is limited by aberrations. For a punctual source, an image without any aberration is obtained if the surface of the mirror is ellipsoidal with the focuses of the ellipsoide at the source and its image (however, due to the non-zero size of the source it is impossible to design an aberration-free mirror). Such an ideal geometry can be approximated by cylindrical surfaces exactly as has been discussed for crystals. The quality of the surface is essential and two parameters are of importance: the roughness and the slope error which are more difficult to obtain low enough (a 


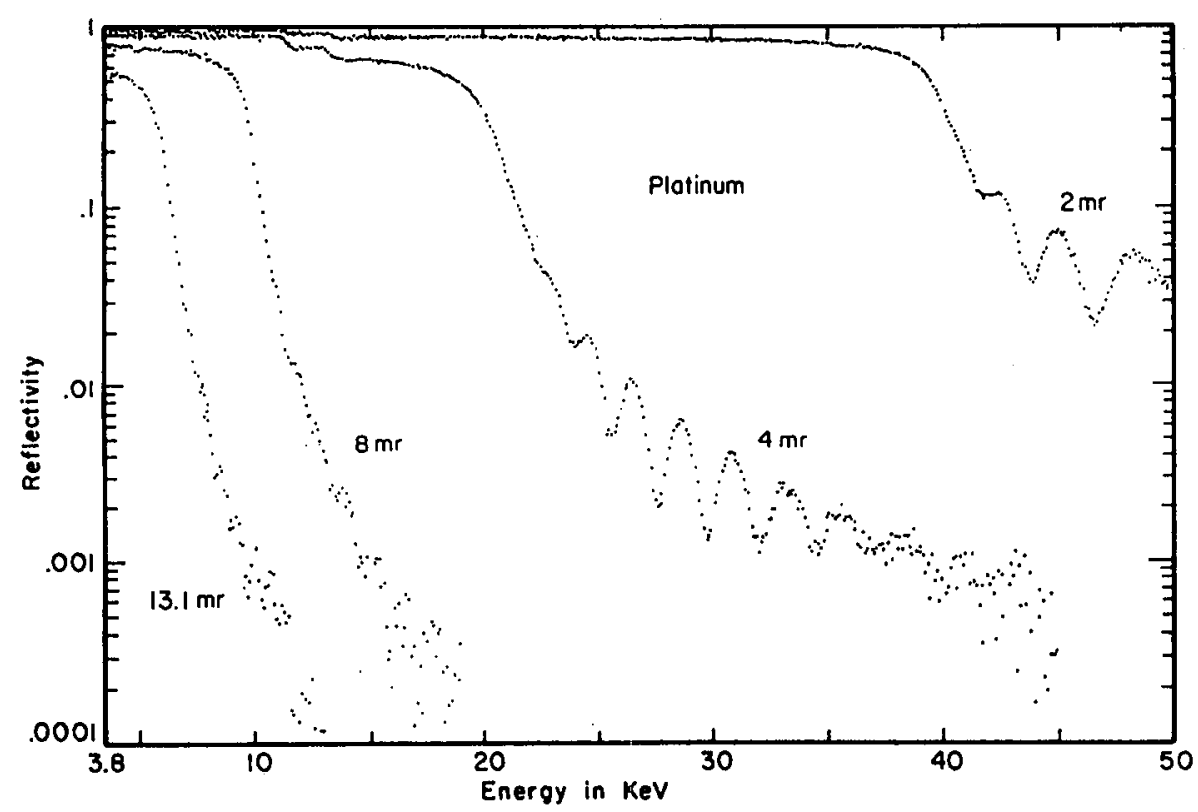

Fig. 15. Reflectivity of a platinum coated mirror as a function of various grazing angles. After [21].

few angströms and arc seconds respectively [22]) for larger surfaces. More details about mirrors can be found in [23].

\section{Polarization}

Before the availability of synchrotron radiation very few people realized $\mathrm{X}$-ray polarized experiments $[24,25]$ because X-ray tubes deliver a low flux of unpolarized $\mathrm{X}$-rays and polarization phenomena in X-ray domain are very specific as is explained below.

Molière showed in 1939 [26] that the dielectric constant of materials and then the index of refraction for $\mathrm{X}$-ray wavelengths is a scalar except near an absorption edge so that, in general, crystals present no birefringence (dependence of the index of refraction on the direction of the electric vector) nor dichroism (dependence of the absorption coefficient, i.e. the imaginary part of the index of refraction on the electric vector).

Molière showed also that crystals are birefringent at Bragg incidence so that a linearly polarized X-ray beam, at Bragg incidence on a perfect crystal, gives an elliptically polarized diffracted beam. This phenomenon was first evidenced experimentally by Skalicky and Malgrange with X-ray tubes [24] and more recently by Golovchenko et al. [27] with synchrotron radiation. At this stage it is necessary to recall some results of X-ray dynamical theory upon which the principles of linear and circular polarizers are based. 


\subsection{Linear polarizers}

$\mathrm{X}$-ray reflectivity depends on polarization because, in two-beam case dynamical theory, equations governing the electrical field for $\sigma$ and $\pi$ polarizations are independent, $\sigma$ and $\pi$ being respectively the component perpendicular and parallel to the scattering plane. The width of the rocking curve for $\pi$-polarized beam decreases proportionally to $\cos 2 \theta$ (as expected from Eq. (4)) tending to zero for Bragg angles going towards $45^{\circ}$. The maximum intensity also decreases when $\cos 2 \theta$ decreases (Fig. 16). Diffraction at a Bragg angle equal to $45^{\circ}$ gives a

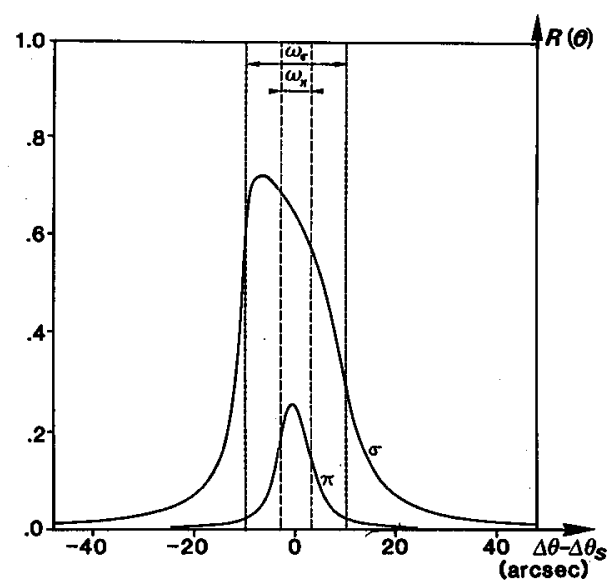

Fig. 16. Rocking curves for $\sigma$ and $\pi$ components for a Si crystal, (111) reflection, $\lambda=3.7 \AA, \theta_{\mathrm{B}}=36.2^{\circ}$.

reflected beam fully linearly polarized with its electric vector perpendicular to the scattering plane. For Bragg angles near $45^{\circ}$, the polarization rate is still good and can be enhanced using two parallel crystals slightly detuned [28].

In the case of transmission, the incident and diffracted waves are coupled in the crystal forming four different Bloch waves: two of them are polarized perpendicularly to the scattering plane ( $\sigma$ wave fields) and the two others in the scattering plane ( $\pi$ wave fields). These Bloch waves are stationary waves whose period is equal to the periodicity of the diffracting planes. For each polarization one wave field (let us call it 1 ) has its nodes on the planes of maximum electrical density in the crystal and is then very much less absorbed than ordinary X-rays, the other one (wave field 2) has its nodes- on the planes of minimum electrical density and is then much absorbed (the absorption coefficients range as follows: $\mu_{\sigma}^{1}<\mu_{\pi}^{1}<\mu_{0}<\mu_{\pi}^{2}<\mu_{\sigma}^{2}$, where $\mu_{0}$ is the ordinary linear absorption coefficient). Furthermore, the index of refraction of these four wave fields are different $\left(n_{\sigma}^{1}>n_{\pi}^{1}>n_{\pi}^{2}>n_{\sigma}^{2}\right)$, these differences depending on the angle of incidence of the incident wave. For a correctly chosen thickness of the sample, only the $\sigma^{1}$ component goes out of the plate and the sample is then a linear polarizer where either the diffracted wave or the transmitted one can be used [29]; using the transmit- 
ted wave one can get a rotation of the polarization vector by rotating the crystal without changing the direction of the beam.

\subsection{Phase-shift retarding plates}

Due to the difference of index of refraction and if the experimental conditions are such that wave field 1 is preponderant it is possible to design phase-shift retarders in transmission geometry $[24,25]$. Howevere, the incident wave has to be very parallel because the difference between $n_{\sigma}$ and $n_{\pi}$ varies rapidly with the angle of incidence. Dmitrienko and Belyakov [30,31] suggested to use perfect crystal in Bragg geometry rotated from Bragg reflection by an angle equal to a few times (5) the width of the rocking curve and to use the transmitted beam. Such a phase-shift retarding plate was realized by Hirano et al. [32].

\subsection{Synchrotron radiation and polarization}

$\mathrm{X}$-ray beams issued from bending magnets sections are mainly polarized in the orbit plane. The polarization depends on the angle of the beam with the orbit plane as is shown in Fig. 17 which indicates the values of the intensity of the components respectively parallel and perpendicular to the orbit plane. For

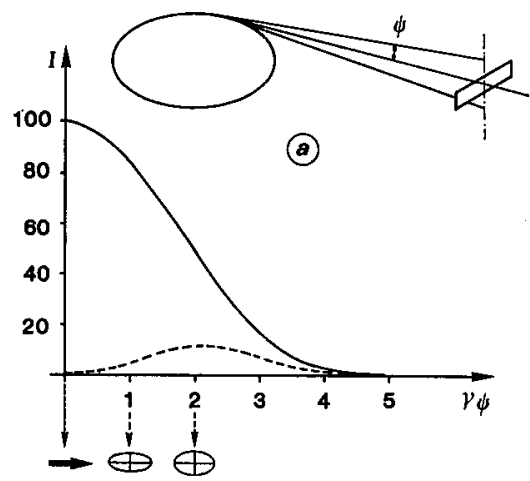

(b)

Fig. 17. Polarization of synchrotron light. Full line: intensity $I_{\mathrm{par}}$ of the component parallel to the orbit plane as a function of the vertical angle $\psi$ for photons with an energy equal to the critical energy $E_{\mathrm{c}} \cdot \gamma=E / m c^{2}$, where $E$ is the energy of the electrons and $m c^{2}-$ their rest mass. Dotted line: intensity $I_{\text {per }}$ of the component perpendicular to the orbit plane. These components have a phase difference of $90^{\circ}$ and the wave is elliptical. The ratio of the ellipse axes $b / a$ is equal to $\left(I_{\mathrm{per}} / I_{\mathrm{par}}\right)^{1 / 2}$.

rays in the orbit plane the beam is linearly polarized; outside the orbit plane it is elliptically polarized because the two components are coherent with a phase difference of $90^{\circ}$. Using horizontal slits which can translate vertically one obtains a beam alternatively linearly or elliptically (left- and right-handed respectively above and below the orbit pland) polarized. 
For X-ray absorption measurements on magnetic or chiral material, for magnetic diffraction or magnetic Compton scattering, circularly (or at least elliptically) polarized beams are essential. Up to now, such experiments have been performed with elliptical light provided by synchrotron beam propagating outside of the orbit plane. progress:

However, two other ways to obtain circularly polarized $\mathrm{X}$-rays are now in

- helical undulators delivering a circularly polarized beam are designed to be placed on third generation synchrotron radiation sources [33]. Let us mention that monochromators should be designed to preserve as much as possible this circular polarization [34].

- production of circularly polarized beams by using phase-plate retarders whose diffracting plane should make an angle of $45^{\circ}$ with the plane formed by the incident beam and the linear electric field.

\section{References}

[1] T. Matsushita, H. Hashizume, in: Handbook on Synchrotron Radiation, Vol. 1, Ed. E.E. Koch, North-Holland, Amsterdam 1983, p. 261.

[2] R. Caciuffo, S. Melone, F. Rustichelli, A. Boeuf, Phys. Rep. 152, 1 (1987).

[3] B.W. Batterman, D.H. Bilderback, in: Handbook on Synchrotron Radiation, Vol. 3, Ed. G. Brown, D.E. Moncton, North-Holland, Amsterdam 1991, p. 105.

[4] M. Krisch, ESRF internal report, ESRF; Grenoble 1988.

[5] A. Freund, in: Synchrotron Radiation in Structural Biology, Ed. R.M. Sweet, A.D. Woodhead, Plenum Press, New York 1989, p. 255.

[6] J.W.M. DuMond, Phys. Rev. 52, 872 (1937).

[7] J.M. Dubuisson, J.M. Dauvergne, C. Depautex, P. Vachette, C.E. Williams, Nucl. Instr. Methods Phys. Res. A 246, 636 (1986).

[8] W.J. Bartels, J. Vac. Sci. Technol. B 1, 338 (1983).

[9] S.M. Heald, Nucl. Instr. Methods Phys. Res. A 266, 457 (1988).

[10] M. Hart, A.R.D. Rodrigues, J. Appl. Crystallogr. 11, 248 (1978).

[11] J.F. Petroff, M. Sauvage, P. Riglet, H. Hashizume, Philos. Mag. A 42, 319 (1980).

[12] C.J. Sparks, J.G.E. Ice, J. Wong, B.W. Batterman, Nucl. Instr. Methods 194, 73 (1982).

[13] B.W. Battermann, L. Berman, Nucl. Instr. Methods 208, 327 (1983).

[14] J. Frouin, Y. Garreau, G. Loupias, D. Raoux, J. Tarbes, Nucl. Instr. Methods Phys. Res. A 266, 484 (1988).

[15] M: Lemonnier, R. Fourme, F. Rousseaux, R. Kahn, Nucl. Instr. Methods 152, 173 (1978).

[16] H. Tolentino, F. Baudelet, E. Dartyge, A. Fontaine, G. Tourillon, Nucl. Instr. Methods Phys. Res. A 289, 307 (1990).

[17] C.J. Sparks, Jr.B.S. Borie, J.B. Hastings, Nucl. Instr. Methods 172, 237 (1980).

[18] M. Krisch, A. Freund, G. Marot, L. Zhang, Nucl. Instr. Methods Phys. Res. A 305, 208 (1991).

[19] H.H. Johann, Z. Phys. 69, 185 (1931). 
[20] T. Johansson, Z. Phys. 82, 507 (1933).

[21] D.H. Bilderback, S. Hubbard, Nucl. Instr. Methods 195, 91 (1982).

[22] G.N. Greaves, C.R.A. Catlow, in: Applications of Synchrotron Radiation, Ed. C.R.A. Catlow, G.N. Grea ves, Blackie, Glasgow 1990.

[23] M.R. Howells, Appl. Opt. 23, 4027 (1980).

[24] P. Skalicky, C. Malgrange, Acta Crystallogr. A 28, 501 (1972).

[25] M. Hart, Philos. Mag. 38, 41 (1978).

[26] G. Molière, Ann. Phys. 35, 272 (1989).

[27] J.A. Golovchenko, B.M. Kincaid, R.A. Levesque, A.E. Meixner, D.R. Kaplan, Phys. Rev. Lett. 57, 202 (1986).

[28] M. Hart, A.R.D. Rodrigues, Philos. Mag. B 40, 149 (1979).

[29] H. Cole, F.W. Chambers, C.G. Wood, J. Appl. Phys. 32, 1942 (1961).

[30] V.E. Dmitrienko, V.A. Belyakov, Sov. Tech. Phys. Lett. 6, 621 (1981).

[31] V.A. Belyakov, V.E. Dmitrienko, Sov. Phys.-Usp. 32, 697 (1990).

[32] K. Hirano, K. Izumi, T. Ishikawa, S. Annaka, S. Kikuta, Jpn. J. Appl. Phys. 30, L407 (1991).

[33] P. Elleaume, ESRF Report SR/ID 88-23; Nucl. Instr. Methods Phys. Res. A 291, 371 (1990).

[34] C. Malgrange, C. Carvalho, L. Braicoviuch, J. Goulon, Nucl. Instr. Methods Phys. Res. A 308, 390 (1991). 